\title{
Translationally invariant treatment of pair correlations in nuclei: I. Spin and isospin dependent correlations
}

\author{
R. Guardiola ${ }^{\text {a }}$, P.I. Moliner ${ }^{\text {a }}$, J. Navarro ${ }^{\text {b }}$, R.F. Bishop ${ }^{\text {c }}$, A. Puente ${ }^{\mathrm{c}}$, \\ Niels R. Walet ${ }^{\mathrm{c}, 1}$ \\ ${ }^{a}$ Dpto. de Física Atómica, Molecular y Nuclear, Universitat de Valencia, Avda. Dr. Moliner 50 , \\ E-46100 Burjassot, Spain \\ b IFIC (Centre Mixt CSIC - Universitat de Valencia), Avda. Dr. Moliner 50, \\ E-46100 Burjassot, Spain \\ ${ }^{c}$ Department of Physics, UMIST, P.O. Box 88, Manchester M60 IQD, UK
}

Received 17 July 1996; revised 2 September 1996

\begin{abstract}
We study the extension of our translationally invariant treatment of few-body nuclear systems to heavier nuclei. At the same time we also introduce state-dependent correlation operators. Our techniques are tailored to those nuclei that can be dealt with in $L S$ coupling, which includes all nuclei up to the shell closure at $A=40$. We study mainly p-shell nuclei in this paper. A detailed comparison with other microscopic many-body approaches is made, using a variety of schematic nuclear interactions. It is shown that our methodology produces very good energies, and presumably also wave functions, for medium mass nuclei.
\end{abstract}

\section{Introduction}

During the last few years there has been an impressive improvement in the methodology used to perform fully microscopic calculations for the ground-state properties of nuclear systems. Most progress has been made for light nuclei, particularly the systems of three and four nucleons, where quite different methods, including Monte Carlo methods [1], Faddeev and Faddeev-Yakubovski methods [2], hyperspherical harmonic

\footnotetext{
${ }^{1}$ E-mail: Niels.Walet@umist.ac.uk. 
expansions [3] and translationally invariant configuration interaction methods [4], have been pushed to a high level of reliability.

Ideally one would like to extend as many of the methods as possible to heavier systems. Unfortunately, however, some of these methods are specially designed for very light systems, and cannot easily be extended to heavier systems. The Green's function Monte Carlo methods have been extended to systems of up to six nucleons and up to eight neutrons, but it is unlikely that such methods will be able to tackle systems with more than a few additional nucleons in the foreseeable future. The hyperspherical harmonic method is also limited in the number of nucleons it can treat, even though the Pisa group has started an ambitious project for p-shell nuclei. Arias de Saavedra et al. [5] have recently investigated the application of the Fermi hypernetted chain (FHNC) method to heavier nuclei, with some apparent success. Another promising approach is one based on a stochastic variational principle [6], but this method is also limited to relatively light nuclei. We should also mention the variational Monte Carlo calculations of ${ }^{16} \mathrm{O}[7,8]$ which have considered realistic interactions. These impressive calculations, however, have used a multiplicative cluster expansion to deal with the operatorial structure of the trial wave function.

In this paper we shall discuss another approach, which was pioneered by us some years ago [4]. This translationally invariant configuration interaction (TICI) method was inspired by the coupled cluster (CC) method. In its lowest-order implementation (CC2) the coupled cluster method consists of using wave functions obtained by acting on a reference state with the exponentiated one- and two-body correlation operators, $\exp \left[S_{1}+S_{2}\right]$. The CC method is most naturally formulated in the occupation-number representation. However, as was to be expected, we found very slow convergence with respect to the cut-off on the single-particle basis in this representation. This led us to consider a linearised form of the CC2 approach, namely the TICI2 method, which also includes pair correlation effects but which can easily be converted to coordinate representation. It has the trial wave function

$$
|\Psi\rangle=\sum_{i<j} f\left(r_{i j}\right)\left|\Psi_{0}\right\rangle .
$$

In order to remove spurious effects due to the centre-of-mass motion the uncorrelated wave function $\left|\Psi_{0}\right\rangle$ must be a harmonic-oscillator shell-model function. In contrast to the standard CC2 method, the TICI2 method is variational, and leads to an upper bound to the ground-state energy.

The simple form of Eq. (1) for the wave function has provided very good estimates of the ground-state energy of ${ }^{4} \mathrm{He}$ for simple Wigner forces [4]. When we revert to occupation-number representation, we find that the ansatz (1) corresponds to the most general shell-model excitation operators containing both one particle-one hole and two particle-two hole components. These components have to be appropriately related so as to ensure the translational invariance.

One obvious improvement of this ansatz is the introduction of three-body correlations, of the form 


$$
|\Psi\rangle=\left(\sum_{i<j} f\left(r_{i j}\right)+\sum_{i<j<k} g\left(r_{i j}, r_{i k}, r_{j k}\right)\right)\left|\Psi_{0}\right\rangle .
$$

Our calculations using this form of trial wave function, with a simple model interaction in ${ }^{4} \mathrm{He}$, produced almost the exact energy for the ground state [4]. As one can see, we have a systematic way of improving the wave function, by adding terms of physical relevance. One may expect that, in medium light nuclei, one must include only up to three- and maybe four-body correlations, in order to get a precise determination of the energy and the structure of the system.

On the other hand, one must also deal with the complex operatorial structure of realistic nucleon-nucleon (NN) interactions, which in turn requires an additional operatorial structure for the correlation operator. At the same time, one also needs to find a way to extend the method to deal with nuclei with a larger number of particles. As is also true for some (but not all) of the other approaches cited above, this extension is trivial, but the underlying computational complexity requires an appreciable effort. We should recall the obvious fact that the expectation value of a two-particle operator, like the NN potential, between states of the type of Eq. (1) requires the evaluation of the expectation value of up to six-body operators. These calculations are large but not prohibitively so.

The dual purpose of this work is to show how this kind of computation may be carried out in practice, and also to investigate the importance of the operatorial structure of the pair correlation. In this paper we are considering simple interactions of the V4 form (central, but spin- and isospin-dependent). We also consider a two-particle correlation operator of the same structure, i.e., with the spin- and isospin-dependent form

$$
f_{i j}=f_{c}\left(r_{i j}\right)+f_{\sigma}\left(r_{i j}\right)\left(\boldsymbol{\sigma}_{i} \cdot \boldsymbol{\sigma}_{j}\right)+f_{\tau}\left(r_{i j}\right)\left(\boldsymbol{\tau}_{i} \cdot \boldsymbol{\tau}_{j}\right)+f_{\sigma \tau}\left(r_{i j}\right)\left(\boldsymbol{\sigma}_{i} \cdot \boldsymbol{\sigma}_{j}\right)\left(\tau_{i} \cdot \boldsymbol{\tau}_{j}\right) .
$$

In Section 2 we discuss the computational problems associated with this form of correlation operator and our way of tackling them. Our scheme goes beyond the typical shell-model methodology, which basically deals with two-body operators. In its current form our calculations are limited to selected nuclei, specifically to those systems which may be described or approximated by spin-isospin saturated wave functions. At the cost of losing the rotational invariance, one may basically deal with $A=4 n$ nuclei, where $n$ is an integer. For some systems (like, e.g., ${ }^{12} \mathrm{C}$ or ${ }^{8} \mathrm{Be}$ ) it is actually better to break the rotational symmetry in favour of a deformed structure. In these nuclei the energy of the deformed state is much lower than that built on a spherical state of the $j j$ coupling scheme. A further restriction on the nuclei we can deal with originates from the fact that our method makes intricate use of $L S$-coupling. This means that our method will not perform well beyond the shell closure at ${ }^{40} \mathrm{Ca}$.

Having discussed our methodology in some considerable detail, we then turn to a comparison of results in Section 3. Our main goal is to gain some insight into both the power of our method and any associated shortcomings. We make a detailed comparison 
with other many-body methods and exact results where available. Finally we draw some conclusions and give an outlook in Section 4.

\section{The evaluation of matrix elements}

In this section we shall show how to calculate matrix elements of a V4-type potential, when the correlations are of the form given in Eq. (3). We present all reductions of the required $A$-body matrix elements by identifying the minimal set of integrals that we are required to calculate. The reduction of spin and isospin degrees of freedom is also discussed, for the case of saturated systems.

The discussion has been limited to the case of the expectation value of the potential, which is the most complex quantity we need to evaluate. A similar, but much less complicated, analysis can be carried out for the kinetic energy operator as well as for the computation of the norm of the pair-correlated state.

\subsection{Diagrammatic analysis}

Let $\left|\Psi_{0}\right\rangle$ represent an independent-particle harmonic-oscillator shell-model wave function for the nucleus under consideration. For the case of the spin-isospin saturated systems in which we are interested, this uncorrelated state may be represented by a single Slater determinant, which is hence fully characterised by the enumeration of the occupied single-particle states. Moreover, given that we are dealing with spin- and isospin-saturated systems, it is only necessary to specify the harmonic oscillator states occupied, and we can ignore spin and isospin for the characterisation of the states. For example, the uncorrelated state for ${ }^{16} \mathrm{O}$ requires the four single-particle states $0 \mathrm{~s}, 0 \mathrm{p}_{+1}$, $0 \mathrm{p}_{0}$ and $0 \mathrm{p}_{-1}$, where the subscript refers to the magnetic quantum number. We can transform to a Cartesian basis where the occupied single-particle states are represented by the three harmonic oscillator quantum numbers $\left(n_{x}, n_{y}, n_{z}\right)$. The reference state for ${ }^{16} \mathrm{O}$ in this basis consists of Slater determinant formed from the four occupied states $(0,0,0),(1,0,0),(0,1,0)$ and $(0,0,1)$.

Consider now the expectation value of the potential energy operator, given by

$$
\langle V\rangle=\left\langle\Psi_{0}\left|\sum_{i<j} f_{L}(i j) \sum_{k<l} V(k l) \sum_{m<n} f_{R}(m n)\right| \Psi_{0}\right\rangle,
$$

where the subscripts $L$ and $R$ identify (for later ease of discussion) the correlation operators at the left-hand side and the right-hand side of the potential operator, respectively. The indices in parentheses label the particles involved in the corresponding correlation or potential operator. When we expand the sums in Eq. (4) there appears a total of $\left(\begin{array}{l}A \\ 2\end{array}\right)^{3}$ terms. One way to classify the individual terms is by the number $N$ of particles involved. This number ranges from 2 (e.g., a term $f_{L}(12) V(12) f_{R}(12)$ ) up to six (e.g., a term $\left.f_{L}(12) V(34) f_{R}(56)\right)$. Moreover, in order to reduce the $A$-particle matrix element to an $N$-particle matrix element it is convenient to decompose the operator of Eq. (4) into a 
sum of symmetric $\mathrm{N}$-body operators. It is very simple to make a diagrammatic analysis of these operators by assigning to all $f$ 's and $V$ 's a line connecting the points specified in their arguments [4]. The topologically distinct diagrams constitute a natural basis for the expansion of the matrix elements.

With some patience one then obtains the decomposition

$$
\sum_{i<j} f_{L}(i j) \sum_{k<l} V(k l) \sum_{m<n} f_{R}(m n)=\sum_{d=1}^{16} \sum_{i_{1}<i_{2}<\cdots<i_{N_{d}}} \mathcal{O}_{i_{1} i_{2} \cdots i_{N_{d}}}^{d},
$$

where the symmetric operators $\mathcal{O}^{d}$ are defined in Table 1 . To clarify the meaning of this table, let us evaluate, for example, the three body part of $f_{L} V f_{R}$. According to the table it can be expanded in the diagrams 2 till 5 as

$$
\begin{aligned}
\left(f_{L} V f_{R}\right)_{i_{1} i_{2} i_{3}}= & \sum_{P}\left[f_{L}\left(i_{1} i_{2}\right) V\left(i_{1} i_{2}\right) f_{R}\left(i_{1} i_{3}\right)+f_{L}\left(i_{1} i_{2}\right) V\left(i_{1} i_{3}\right) f_{R}\left(i_{1} i_{3}\right)\right. \\
& \left.+f_{L}\left(i_{1} i_{2}\right) V\left(i_{1} i_{3}\right) f_{R}\left(i_{1} i_{2}\right)+f_{L}\left(i_{1} i_{2}\right) V\left(i_{1} i_{3}\right) f_{R}\left(i_{2} i_{3}\right)\right],
\end{aligned}
$$

where the sum over permutations $P$ includes the six permutations of the triplet $\left(i_{1} i_{2} i_{3}\right)$. In other cases there may be restrictions on the permutations, as mentioned in the table. The number of permutations $P_{d}$ of a given diagram under these restriction is listed in the last column of the table. This allows the enumeration of the diagrams to be checked, since they must satisfy the equality

$$
\left(\begin{array}{c}
A \\
2
\end{array}\right)^{3}=\sum_{d=1}^{16} P_{d}\left(\begin{array}{c}
A \\
N_{d}
\end{array}\right)
$$

where $d$ refers to a given diagram, and $N_{d}$ is the number of particles involved in that diagram.

After this classification, the expectation value of a given diagram between Slater determinantal wave functions is reduced to

$$
\begin{aligned}
& \left\langle\Psi_{0}\left|\sum_{i_{1}<\cdots<i_{N_{d}}} \mathcal{O}_{i_{1} \cdots i_{N_{d}}}^{d}\right| \Psi_{0}\right\rangle \\
& =\frac{1}{N_{d} !} \sum_{i_{1} \cdots i_{N_{d}}=1}^{A}\left\langle\psi_{i_{1}}(1) \cdots \psi_{i_{N_{d}}}\left(N_{d}\right)\left|\mathcal{O}_{1 \cdots N_{d}}^{d}\right| \sum_{P} \epsilon_{P} \psi_{P_{i_{1}}}(1) \cdots \psi_{P_{N_{N_{d}}}}\left(N_{d}\right)\right\rangle .
\end{aligned}
$$

This equation requires further explanation. The single-particle states are represented by the wave functions $\psi_{i}(j)$, which also includes a spinor and an isospinor. Here the subscript $i$ refers to the single particle state, and the index in parenthesis specifies the particle involved. The right-hand side of the equation also includes a sum over permutations of the single-particle state labels, without any restriction. Note that the sum over single particle states extends over all occupied orbitals.

A very important reduction in the computation is related to a further symmetry of Eq. (6), and refers to the sum of permutations (not explicitly written in this equation) 
Table 1

The topologically distinct diagrams for potential matrix elements. The column labelled $d$ gives a unique label for each diagram, $N_{d}$ specifies the number of particles involved. The column labelled $f_{L}$ gives the particle labels $(i j)$ of the left-hand side correlation function, $V$ those $(k l)$ of the potential and $f_{R}$ those $(m n)$ of the right-hand side correlation. The diagrams must be symmetrised with respect to the particle labels $i_{1} \cdots i_{N_{d}}$ involved, but with some restrictions to avoid overcounting. These restrictions are given by the order in which the labels appear on the functions $f_{L, R}$ and $V$. The number of permutations $P_{d}$ under these restrictions is given in the last column

\begin{tabular}{rllllll}
\hline$d$ & $N_{d}$ & $\begin{array}{l}f_{L} \\
i j\end{array}$ & $\begin{array}{l}V \\
k l\end{array}$ & $\begin{array}{l}f_{R} \\
m n\end{array}$ & $\begin{array}{l}\text { Restrictions } \\
\text { on permutations }\end{array}$ & $P_{d}$ \\
\hline 1 & 2 & $i_{1} i_{2}$ & $i_{1} i_{2}$ & $i_{1} i_{2}$ & $i<j$ & 1 \\
2 & 3 & $i_{1} i_{2}$ & $i_{1} i_{2}$ & $i_{1} i_{3}$ & none & 6 \\
3 & & $i_{1} i_{2}$ & $i_{1} i_{3}$ & $i_{1} i_{3}$ & none & 6 \\
4 & & $i_{1} i_{2}$ & $i_{1} i_{3}$ & $i_{1} i_{2}$ & none & 6 \\
5 & & $i_{1} i_{2}$ & $i_{1} i_{3}$ & $i_{2} i_{3}$ & none & 6 \\
6 & \multirow{2}{*}{4} & $i_{1} i_{2}$ & $i_{1} i_{2}$ & $i_{3} i_{4}$ & $i<j, m<n$ & 6 \\
7 & & $i_{1} i_{2}$ & $i_{3} i_{4}$ & $i_{3} i_{4}$ & $i<j, m<n$ & 6 \\
8 & & $i_{1} i_{2}$ & $i_{3} i_{4}$ & $i_{1} i_{2}$ & $i<j, k<l$ & 6 \\
9 & & $i_{1} i_{2}$ & $i_{1} i_{3}$ & $i_{3} i_{4}$ & none & 24 \\
10 & & $i_{1} i_{2}$ & $i_{1} i_{3}$ & $i_{1} i_{4}$ & none & 24 \\
11 & & $i_{1} i_{2}$ & $i_{3} i_{4}$ & $i_{1} i_{3}$ & none & 24 \\
12 & & $i_{1} i_{2}$ & $i_{2} i_{3}$ & $i_{1} i_{4}$ & none & 24 \\
13 & 5 & $i_{1} i_{2}$ & $i_{3} i_{4}$ & $i_{1} i_{5}$ & $k<l$ & 60 \\
14 & & $i_{1} i_{2}$ & $i_{1} i_{3}$ & $i_{4} i_{5}$ & $m<n$ & 60 \\
15 & & $i_{1} i_{2}$ & $i_{3} i_{4}$ & $i_{3} i_{5}$ & $i<j$ & 90 \\
\hline 16 & 6 & $i_{1} i_{2}$ & $i_{3} i_{4}$ & $i_{5} i_{6}$ & $i<j, k<l, m<n$ & \\
\hline
\end{tabular}

which enter into the definition of $\mathcal{O}^{d}$. It turns out that each of these permutations gives the same contribution to the matrix element, so that it is not necessary to evaluate all permutations separately. It is sufficient to consider one of the diagrams contributing to $\mathcal{O}^{d}$, and multiply the resulting expectation value by the number of permutations of the diagram given in the last column of Table 1.

\subsection{The spin and isospin degrees of freedom}

To evaluate a given matrix element, Eq. (6), one must sum over all occupied singleparticle states. In the case of spin-isospin saturated systems, the index labelling the single-particle states may be split into three pieces,

$$
i \equiv\left(i^{s}, i^{\sigma}, i^{\tau}\right),
$$

where $s, \sigma$ and $\tau$ refer to space, spin and isospin, respectively. The sum over all occupied states is equivalent to a sum over harmonic-oscillator states, together with a replacement of all products of spin and isospin operators by their respective traces.

The computation of the trace requires a further reduction, since it interferes with the calculation of permutations. Each of the permutations appearing in the $N$-particle state 
of Eq. (6) can be split into three independent permutations, as can be seen from the pair-wise exchange

$$
(i j) \equiv\left(i^{s} j^{s}\right)\left(i^{\sigma} j^{\sigma}\right)\left(i^{\tau} j^{\tau}\right)
$$

The pairwise permutations referring to spin and isospin indices can be implemented by having familiar spin and isospin exchange operators, $P_{i j}^{\sigma}$ and $P_{i j}^{\tau}$ act on the wave function. Thus the spin and isospin part of the permutation $P, P^{\sigma}$ and $P^{\tau}$, can be written as products of exchange operators, which can be included inside the trace over spin and isospin.

We thus obtain the matrix element

$$
\begin{aligned}
& \left\langle\Psi_{0}\left|f_{L}(i j) V(k l) f_{R}(m n)\right| \Psi_{0}\right\rangle \\
& =\sum_{p q r} \sum_{i_{1}^{s} \cdots i_{N}^{s}=1}^{A / 4} \sum_{P} \epsilon_{P} \operatorname{Tr}\left(\Theta_{i j}^{p} \Theta_{k l}^{q} \Theta_{m n}^{r} P_{i_{1}^{\sigma} \cdots i_{N}^{\sigma}}^{\sigma} P_{i_{1}^{\tau} \ldots i_{N}^{\tau}}^{\tau}\right) \\
& \quad \times\left\langle\phi_{i_{1}^{s}}(1) \cdots \phi_{i_{N}^{s}}(N)\left|f_{L}^{p}\left(r_{i j}\right) V^{q}\left(r_{k l}\right) f_{R}^{r}\left(r_{m n}\right)\right| \phi_{P_{1}^{s}}(1) \cdots \phi_{P i_{N}^{s}}(N)\right\rangle .
\end{aligned}
$$

Here the $\phi$ 's are the spatial (harmonic oscillator) parts of the single-particle wave functions $\psi$ in Eq. (6). Since all spin and isospin sub-states are filled, each harmonic oscillator state can be occupied at most four times. Both the left and right correlation operators have been expanded in a basis of operators,

$$
f(i j)=\sum_{k} f^{k}\left(r_{i j}\right) \Theta^{k}(i j)
$$

with a similar expansion for the potential operator. A quite convenient basis for a V4 form is the set of spin and isospin exchange operators,

$$
\Theta^{1}(i j)=1, \Theta^{2}(i j)=P_{i j}^{\sigma}, \Theta^{3}(i j)=P_{i j}^{\tau}, \Theta^{4}(i j)=P_{i j}^{\sigma} P_{i j}^{\tau}
$$

Since the spin and isospin permutation operators can also be written as product of exchange operators, we now have to evaluate the trace of a long string of exchange operators.

\subsection{The computation of traces}

For the case of a V4 set of operators, both for correlations and interaction, the calculation of the traces is rather simple. For an $N$-particle subsystem, a given spin (or isospin) state may be represented by a column vector of $2^{N}$ components, where a given component defines all orientations of the $N$ spins. A simple representation is obtained by assigning a binary sequence to a given set $\left[\sigma_{1}, \sigma_{2}, \ldots, \sigma_{N}\right]$ of spins:

$$
\mathcal{N}=\sigma_{1}+2 \sigma_{2}+4 \sigma_{3}+\cdots+2^{(N-1)} \sigma_{N}
$$

where we use $\sigma_{i}=0$ for spin up and 1 for spin down. 
With this notation, a spin exchange operator is represented by a $2^{N} \times 2^{N}$ matrix with only one non-zero element per row and column. This facilitates the storage of matrices, the multiplication of exchange operators and the computation of the trace.

We have to evaluate a large number of traces, since we have four components for the left correlation, the potential and the right correlation, as well as the 720 permutations of a six-particle subsystem, leading to a total of $720 \times 4^{3}=46080$. With the representation introduced above it is however rather straightforward - and very efficient - to write a computer program that evaluates all these traces.

\subsection{Spatial integrals}

In order to make the calculation manageable we expand the correlation functions in a set of Gaussians,

$$
f^{p}(r)=\sum_{i} c_{i}^{p} \exp \left(-\beta_{i} r^{2}\right)
$$

a technique that has been proven to give an excellent representation of the correlation function $f$ if we include negative as well as positive values of $\beta$ [4]. This is well matched to the use of harmonic oscillator single-particle states, since the required space integrals can be shown to be of the form of the product of an exponential of a positivedefinite quadratic form with a polynomial.

If the potential is a combination of Gaussians, like the S3 interaction of Afnan and Tang [9], it may also be absorbed into the quadratic form in the exponential. Then, the required integrals can be computed by using a recurrence relation [10].

For other algebraic forms, we can follow two routes. One is to fit a set of Gaussians to the potential, and apply the technique discussed above. We choose to follow another route [10], where we perform all integrals apart from those involving the coordinates in the potential by the technique sketched above. The remaining one-dimensional integral may then be computed by means of a suitable numerical method.

\subsection{Solution}

Since the different components in the decomposition of Eq. (11) of the correlation function are not orthogonal, the expectation value of the energy can be given in the form

$$
E\left(\left\{c_{i}^{p}\right\}\right)=\frac{\sum_{(i p),(j q)} c_{i}^{p *} \mathcal{H}_{i j}^{p q} c_{j}^{q}}{\sum_{(i p),(j q)} c_{i}^{p *} \mathcal{N}_{i j}^{p q} c_{j}^{q}},
$$

where $c_{i}^{p}$ is the coefficient multiplying the $i$ th Gaussian in the correlation function attached to operator $p$. If we now optimise with respect to $c_{i}^{p *}$ we end up with a generalised eigenvalue problem 
$\sum_{(j q)} \mathcal{H}_{i j}^{p q} c_{j}^{q}=\lambda \sum_{(j q)} \mathcal{N}_{i j}^{p q} c_{j}^{q}$

The lowest eigenvalue $\lambda$ gives the approximation for the ground-state energy.

\subsection{The helium case}

The four-nucleon system deserves a special treatment, since the decomposition of the two-particle correlation introduced in Eq. (8) is over-complete for this special nucleus. This fact is related to the special form of the uncorrelated state, which is fully space-symmetric, so that the action of the operators tied to the correlation function is strongly simplified. In particular, the action of the spin-isospin exchange operator $P_{i j}^{\sigma} P_{i j}^{\tau}$ is equivalent to -1 , and the action of $P_{i j}^{\sigma}$ is the same as the action of $-P_{i j}^{\tau}$. Clearly, one should limit the general form of the correlation so as not to create duplicate basis states, since this will give rise to problems when solving the generalised eigenvalue problem of Eq. (13). Specifically, the state-dependent correlation of the $\mathrm{V} 4$ type for ${ }^{4} \mathrm{He}$ should include only the central scalar (Wigner) and spin-exchange (Bartlett) pieces. Equivalently, one may include two separate correlation functions, one for the singlet channels and one for the triplet channels.

\section{Results}

Using our technology we have set out to calculate the binding energy of ${ }^{4} \mathrm{He},{ }^{8} \mathrm{Be}$, ${ }^{12} \mathrm{C}$ and ${ }^{16} \mathrm{O}$. The results are presented in Table 2 . In order to allow for the best possible comparison with existing results in the literature we have investigated the Brink-Boeker B1 potential [11], the Afnan-Tang S3 potential [9], the modified S3 (MS3) potential [12], and the Malfliet-Tjon MT I/III and MT V potentials [13]. We have optimised the value of $\alpha$, the inverse length-scale of the harmonic oscillator, for the case of the state-independent (SI), purely central (scalar) correlations, i.e., where we restrict the expansion of Eq. (8) to the $k=1$ term only. We then performed a calculation with the full state-dependent (SD) V4 type correlation operator, keeping the value of $\alpha$ fixed. The difference between the results from the use of a central state-independent (SI) and the use of a state-dependent (SD) correlation operator is thus probably slightly underestimated. Our calculations for ${ }^{8} \mathrm{Be}$ and ${ }^{12} \mathrm{C}$ were performed using a non-spherical reference state (in Cartesian notation)

$$
\left|\Psi_{0}\right\rangle=\left|(0,0,0)^{4}(0,0,1)^{4}\right\rangle
$$

for ${ }^{8} \mathrm{Be}$ and a similar state,

$$
\left|\Psi_{0}\right\rangle=\left|(0,0,0)^{4}(1,0,0)^{4}(0,1,0)^{4}\right\rangle
$$

for ${ }^{12} \mathrm{C}$. We recall that we can only use states of this form since we work so as to have spin and isospin saturation, within an $L S$ coupling scheme. We have broken the 
Table 2

Energies of p-shell nuclei for the five interactions considered, B1, S3, MS3, MT I/III and MT V. The column labelled SI corresponds to a purely scalar (state-independent) pair correlation, and the column labelled SD corresponds to a (state-dependent) pair correlation of V4 type

\begin{tabular}{|c|c|c|c|}
\hline Nucleus & $\alpha\left(\mathrm{fm}^{-1}\right)$ & $E_{\mathrm{Si}}(\mathrm{MeV})$ & $E_{\mathrm{SD}}(\mathrm{MeV})$ \\
\hline \multicolumn{4}{|c|}{ B1 interaction } \\
\hline${ }^{4} \mathrm{He}$ & 0.729 & -37.86 & -37.86 \\
\hline${ }^{8} \mathrm{Be}$ & 0.595 & -49.18 & -61.30 \\
\hline${ }^{12} \mathrm{C}$ & 0.595 & -84.91 & -103.93 \\
\hline${ }^{16} \mathrm{O}$ & 0.602 & -145.94 & -167.30 \\
\hline \multicolumn{4}{|c|}{ S3 interaction } \\
\hline${ }^{4} \mathrm{He}$ & 0.717 & -25.41 & -28.19 \\
\hline${ }^{8} \mathrm{Be}$ & 0.633 & -34.15 & -44.26 \\
\hline${ }^{12} \mathrm{C}$ & 0.671 & -71.14 & -87.78 \\
\hline${ }^{16} \mathrm{O}$ & 0.707 & -141.64 & -164.88 \\
\hline \multicolumn{4}{|c|}{ MS3 interaction } \\
\hline${ }^{4} \mathrm{He}$ & 0.713 & -25.41 & -27.99 \\
\hline${ }^{8} \mathrm{Be}$ & 0.582 & -26.26 & -37.30 \\
\hline${ }^{12} \mathrm{C}$ & 0.588 & -46.22 & -62.99 \\
\hline${ }^{16} \mathrm{O}$ & 0.596 & -85.56 & -105.64 \\
\hline \multicolumn{4}{|c|}{ MT I/III interaction } \\
\hline${ }^{4} \mathrm{He}$ & 0.741 & -29.45 & -30.81 \\
\hline${ }^{8} \mathrm{Be}$ & 0.660 & -46.67 & -52.67 \\
\hline${ }^{12} \mathrm{C}$ & 0.698 & -99.05 & -109.04 \\
\hline${ }^{16} \mathrm{O}$ & 0.744 & -194.10 & -207.52 \\
\hline \multicolumn{4}{|c|}{ MT V interaction } \\
\hline${ }^{4} \mathrm{He}$ & 0.741 & -29.45 & -29.45 \\
\hline${ }^{8} \mathrm{Be}$ & 0.869 & -129.25 & -130.23 \\
\hline${ }^{12} \mathrm{C}$ & 0.997 & -425.99 & -429.44 \\
\hline${ }^{16} \mathrm{O}$ & 1.078 & -966.65 & -973.67 \\
\hline
\end{tabular}

rotation symmetry in these cases, and we no longer get a strict estimate for the groundstate energy, but for the slightly higher intrinsic energy - a weighted average of the energies of the $J=0,2,4$ states comprising the ground-state band. Nevertheless, such an estimate is preferable to one using a spherical wave function (e.g., $\left|\left(0 \mathrm{~s}_{1 / 2}\right)^{4}\left(0 \mathrm{p}_{3 / 2}\right)^{8}\right\rangle$ for the ground state of ${ }^{12} \mathrm{C}$ ). This can be shown trivially from a naive calculation of the ${ }^{12} \mathrm{C}$ nucleus using harmonic oscillator states and the $\mathrm{B} 1$ potential, without correlations. The energies in the spherical state $\left|\left(0 \mathrm{~s}_{1 / 2}\right)^{4}\left(0 \mathrm{p}_{3 / 2}\right)^{8}\right\rangle$ and in the non-spherical state $\left|(0,0,0)^{4}(1,0,0)^{4}(0,1,0)^{4}\right\rangle$ are -43.11 and $-59.04 \mathrm{MeV}$, respectively, while a shellmodel (or configuration interaction) diagonalisation of the Hamiltonian in the 0p-shell gives $-62.07 \mathrm{MeV}$. Thus an uncorrelated estimate based on the deformed state appears to be almost optimal. Note that the energy may be lowered still further by using a deformed single particle basis, i.e., by using different harmonic oscillator parameters along the different axes. 
Table 3

A comparison of the binding energies (in MeV) of ${ }^{4} \mathrm{He}$ and ${ }^{16} \mathrm{O}$ using various techniques with the MalflietTjon MT V potential. TICI2 is the method presented in this paper, VMC are various variational Monte Carlo calculations, GFMC and DMC are fixed-node Green's function and diffusion Monte Carlo methods, FHNC is the Fermi hypernetted chain method based on a Jastrow wave function, IDEA is the so-called integro-differential equation approach, and SVM is the stochastic variational method

\begin{tabular}{lll}
\hline & ${ }^{4} \mathrm{He}$ & ${ }^{16} \mathrm{O}$ \\
\hline TICI2(SI) & 29.45 & 966.65 \\
TICI2(SD) & 29.45 & 973.67 \\
VMC & & $1024 \pm 5[14]$ \\
& & $1103 \pm 1[15]$ \\
& & $1138.5 \pm 0.2[16]$ \\
GFMC [14] & $31.3 \pm 0.2$ & $1194 \pm 20$ \\
DMC & $31.32 \pm 0.02[17]$ & $1189 \pm 1[16]$ \\
FHNC/0 [18] & & $987 / 1152$ \\
FHNC//0 [19] & & $1059 / 1055$ \\
IDEA [20] & $30.7-31.2$ & $1021-1027$ \\
SVM [6] & 31.360 & \\
\hline
\end{tabular}

The effects of improving the correlations are very interesting. Note that for the B1 and MT V interactions the introduction of SD-type correlations does not make a difference for ${ }^{4} \mathrm{He}$. This is due to the fact that these interactions contain only purely central scalar (Wigner) and space-exchange (Majorana) terms, and hence do not couple to the spin-dependent piece of the correlation operator in ${ }^{4} \mathrm{He}$. Even though there is no spin-dependent part to these potentials, spin-dependent correlations do play a role for all heavier nuclei, so long as the uncorrelated state is not fully space-symmetric. In the $\mathrm{B} 1$ case the difference is over $20 \mathrm{MeV}$ for ${ }^{16} \mathrm{O}$. The effect is smaller for the MT V potential, but this interaction is very pathological - it does not saturate nuclear matter. For the S3, MS3 and MT I/III interactions we find that the effect of the additional correlations is in the 10 to $20 \mathrm{MeV}$ range for ${ }^{16} \mathrm{O}$, an important difference.

In Fig. 1 we show the correlation functions for the four p-shell nuclei. Apart from the case of ${ }^{8} \mathrm{Be}$ the central scalar correlation function changes little from the SI to SD calculation. Indeed, one could almost keep this one fixed and only vary the additional three. This may be due to some kind of perturbation argument: in general the spinand/or isospin-dependent correlation functions are a lot smaller than the central one. The spin and isospin correlation functions are almost equal in magnitude but opposite in sign. There is no obvious argument why this should be so, but it may be due to some approximate persistence of the corresponding exact relation in the case of ${ }^{4} \mathrm{He}$.

In order to be able to judge the power of our technology we compare several different methodologies for the purely scalar MT V potential in Table 3. For ${ }^{4} \mathrm{He}$ our results compare favourably with the Green's function and diffusion Monte Carlo results, both of which have now been superseded by various highly accurate calculations, including one based on the stochastic variational principle (see Ref. [6] and references in that paper). More surprising is the comparison for ${ }^{16} \mathrm{O}$. For the MT V potential, with its extremely strong binding, ${ }^{16} \mathrm{O}$ is a high-density system. Our calculations with only two- 

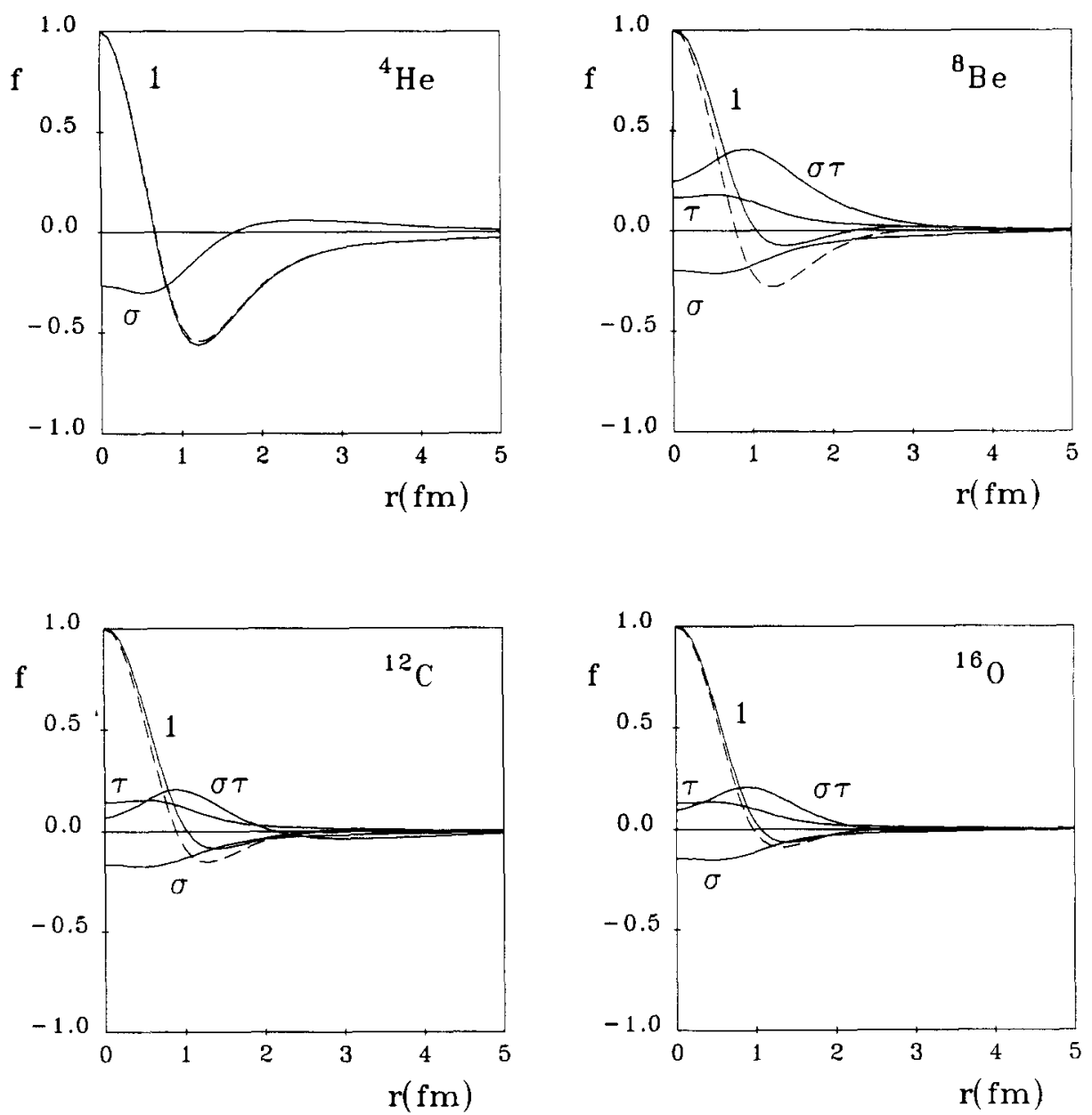

Fig. 1. The calculated correlation functions in the case of the MS3 potential. Dashed lines correspond to purely central scalar SI correlations and solid lines to SD correlations of V4 type. In the latter case the labels 1, $\sigma, \tau$ and $\sigma \tau$ correspond to the terms $k=1,2,3$ and 4 in the parametrisation of Eqs. (8) and (9). As discussed in the text, for helium only two such functions should be used.

body correlations are able to reproduce over $80 \%$ of the binding energy ( $974 \mathrm{MeV}$ for $\mathrm{TICI}$ (SD) as compared to $1189 \mathrm{MeV}$ for the very accurate DMC result). Inclusion of state-independent three-body correlations in our calculation - a relatively straightforward extension - should probably be able to give a highly accurate answer.

The TICI2 method also appears to be competitive with several of the methods based on Jastrow-correlated non-interacting wave functions of a single Slater determinant form, where the Jastrow correlation operator is a product over all pairs of particles of a scalar (state-independent) pair function of the interparticle distance only. The many-body energy expectation value for such trial Jastrow wave functions cannot be calculated analytically, but may be calculated within controlled error bands using variational Monte Carlo (VMC) techniques. Each of the three VMC results cited in Table 3 is of this 
form, with the differences between them resulting from different choices of singleparticle wave functions and from different forms of the Jastrow correlation function over which the variational search is made. These are discussed in more detail below. As an alternative to the stochastically exact VMC calculations of the energy expectation value, the various Fermi hypernetted chain (FHNC) approximations $[5,18,19]$ represent massively resummed cluster expansions of the same quantity, in which some cluster terms (of chain or ladder form) are summed to all orders but various so-called elementary diagrams are neglected or approximated. The resulting FHNC approximants for the ground-state energy are no longer strict variational bounds, insofar as the higher-order terms have been neglected.

Firstly, the VMC result of $1024 \pm 5 \mathrm{MeV}$ for the binding of ${ }^{16} \mathrm{O}$ with the MT V potential from Ref. [14] uses single-particle wave functions generated by a WoodsSaxon potential and a scalar Jastrow correlation function obtained from a constrained Euler-Lagrange optimisation of the second order cluster expansion approximation to the energy functional. We note that the corresponding result from the lowest order FHNC calculation, in the formulation of Fantoni and Rosati, the so-called FHNC/0 method [18] in which the elementary diagrams are neglected completely, using the same singleparticle wave functions and Jastrow correlation function, is $987 \mathrm{MeV}$. Secondly, the VMC result of $1103 \pm 1 \mathrm{MeV}$ from Ref. [15] for the ${ }^{16} \mathrm{O}$ binding energy employs harmonic oscillator single-particle wave functions and a Jastrow correlation function of Gaussian form, $f(r)=1+a \exp \left(-r^{2} / b^{2}\right)$, with the oscillator length parameter and the constants $a$ and $b$ optimised. Finally, the similar VMC result of $1138.5 \pm 0.2$ from Ref. [16] uses optimised harmonic oscillator single-particle wave functions and a Jastrow correlation function of the form $f(r)=\exp \left(-a \mathrm{e}^{-b r}\right)$.

We note that a so-called FHNC//0 calculation for ${ }^{16} \mathrm{O}$ using the MT V potential has been performed by Krotscheck [19]. By using the best possible choices for both the single particle wave functions and the Jastrow correlation function (i.e., by solving the corresponding Euler-Lagrange equation which arise by making the FHNC//0 energy functional stationary) he obtains a value of $1059 \mathrm{MeV}$ for the binding energy. Furthermore, by optimising only the Jastrow correlation function via his Euler-Lagrange approach, and using single-particle wave functions obtained from a Woods-Saxon potential which reproduces the r.m.s. radius of the GFMC calculation of Ref. [14], he obtains the only very slightly worse value of $1055 \mathrm{MeV}$. He estimates, using the $\mathrm{FHNC} / \mathrm{C}$ approximation that the contribution from the neglected exchange diagrams is $14 \mathrm{MeV}$. We also note that by employing precisely the same nearly optimal Woods-Saxon single-particle wave functions, but with a Jastrow correlation function obtained from a constrained minimisation of the two-body cluster expansion approximation to the energy functional, a corresponding FHNC/0 calculation [18] gives a ${ }^{16} \mathrm{O}$ binding energy of $1152 \mathrm{MeV}$. This difference of nearly $100 \mathrm{MeV}$ is presumably a measure of the uncertainty in the FHNC/0 results, in the light of the other results cited. It seems rather unlikely that the corresponding VMC calculation for this same wave function will give such a large value for the binding energy. It would thus appear that, at least for this very unrealistic potential, the latter FHNC/0 result does not provide a true variational estimate. We defer 
Table 4

A comparison of the binding energies (in $\mathrm{MeV}$ ) of ${ }^{12} \mathrm{C}$ and ${ }^{16} \mathrm{O}$ using various techniques with the Brink-Boeker $\mathrm{Bl}$ potential. For a description of the methods see the caption of the previous table. FAHT is a cluster expansion

\begin{tabular}{lcl}
\hline & ${ }^{12} \mathrm{C}$ & ${ }^{16} \mathrm{O}$ \\
\hline TICI2(SI) & $84.9]$ & 145.94 \\
TICI2(SD) & 103.93 & 167.30 \\
VMC [21] & $82.9 \pm 0.2$ & $150.9 \pm 0.3$ \\
FHNC/0 & & $168.2^{\mathrm{a}} / 167.2^{\mathrm{b}}[18]$ \\
& $68.9^{\mathrm{c}}[5]$ & $\left.161.7^{\mathrm{c}} \mid 5\right]$ \\
FHNC-1 & $63.7^{\mathrm{c}}[5]$ & $150.4^{\mathrm{a}} / 152.4^{\mathrm{b}}[18]$ \\
& 82.3 & $151.4^{\mathrm{c}}[5]$ \\
FAHT-4 [21] & $79.8-80.1$ & 148.6 \\
IDEA [20] & $164.4-165.2$ \\
\hline
\end{tabular}

a State-independent Gaussian, $L S$ coupling.

b State-independent 2-body Euler-Lagrange, $L S$ coupling.

c State-independent 2-body Euler-Lagrange, $j j$ coupling.

to Section 4 a further discussion of the FHNC results.

The reason for investigating the MT V potential is that quite a few results are available, including very good GFMC and DMC calculations. However, we also investigate the B1 and MS3 potentials which have more sensible saturation properties, but for which no "exact" results are available to act as a benchmark for the calculations. B1 is a Wignerplus-Majorana potential, whereas MS3 is a full V4-type potential. It is interesting to look at both potentials in turn, since they highlight different aspects of the use of V4-type correlations.

For the Brink-Boeker $\mathrm{B} 1$ potential we present results in Table 4 for the nuclei ${ }^{12} \mathrm{C}$ and ${ }^{16} \mathrm{O}$, the only nuclei for which several other results are available. For ${ }^{12} \mathrm{C}$ we must distinguish between calculations like our own that studied the intrinsic state for the ground-state band and those that start from a spherical state. Unfortunately, there are no GFMC or DMC results available for either nucleus with the B1 potential, and we can only compare with the results from other many-body techniques. All of the other results in Table 4, except those from IDEA, are based on a Jastrow form of the wave function, again using a scalar (state-independent) correlation operator. The results labelled FAHT$n$ are from an $n$th order cluster expansion of the Jastrow energy functional in the socalled factorised Aviles-Hartogh-Tolhoek decomposition [22], which is multiplicative Van Kampen-like version of the original additive Ursell-like AHT version.

Our SI calculation for ${ }^{12} \mathrm{C}$ results in slightly better values for the binding energy than the VMC and FAHT calculations. Note, however, that Jastrow calculations with anisotropic $\mathrm{HO}$ wave functions result in an increase of the binding energy of around $10 \mathrm{MeV}(92.3 \pm 0.3 \mathrm{MeV}$ with the VMC method of Ref. [21]). However, when we include state-dependent (SD) correlations in our TICI2 calculation we find an increase in binding of nearly $20 \mathrm{MeV}$. Bearing in mind that our results are fully variational it is clear that our TICI2(SD) calculation is better than any of the other results shown for ${ }^{12} \mathrm{C}$ with the $\mathrm{B} 1$ potential. We note also that the results based on a spherical assumption 
give appreciably less binding for ${ }^{12} \mathrm{C}$. As we have discussed previously, this is really a trivial effect, which arises primarily at the level of the uncorrelated wave function. This is particularly so for the FHNC results [5] based on a $j j$ coupling scheme. We show results for both the lowest-order FHNC/0 approximation of the Fantoni-Rosati scheme, in which all elementary diagrams are neglected, and for their FHNC-1 approximation in which the first-order elementary exchange diagram has been included. Such diagrams are clearly likely to be of more importance for potentials of the Bl type with an appreciable Majorana component than for purely scalar potentials of the MT V type. The effect of their inclusion is seen to reduce the binding. The discrepancy between the VMC result [21] of $82.9 \pm 0.2 \mathrm{MeV}$ and the FHNC-1 result [5] in the $j j$ basis of $63.7 \mathrm{MeV}$ is surprisingly large. We note, however, that the state-independent Jastrow calculations in Ref. [5] were obtained in a calculation which included the Coulomb force, by minimisation of the energy at the second order of the cluster expansion. The results shown in Table 4 have been obtained by subtracting out the quoted values of the Coulomb energy to the binding energies given in Ref. [5]. Clearly, it is conceivable that had the minimisation been done without the inclusion of the Coulomb force, slightly improved results might have been obtained. We also note that the calculations in Ref. [5] were performed with Woods-Saxon single-particle wave functions, thereby making it impossible to remove centre-of-mass effects exactly.

In the case of ${ }^{16} \mathrm{O}$ with the $\mathrm{B} 1$ potential we find similar results to the ${ }^{12} \mathrm{C}$ case. Again our TICI2(SI) calculation gives a good result, and the improvement obtained by allowing for state-dependent correlations leads to what is possibly the best (and certainly the variationally most reliable) result now available for this case. The difference in binding between our SI and SD calculations of over $20 \mathrm{MeV}$ is clearly very appreciable. We note that the FHNC/0 and FHNC-1 results for ${ }^{16} \mathrm{O}$ have been done in both $L S$ [18] and $j j$ [5] coupling schemes. For the former case we cite results using Jastrow correlation functions both parametrised as a Gaussian form, $f(r)=1+a \exp \left(-r^{2} / b^{2}\right)$, and from a constrained Euler-Lagrange minimisation of the second-order cluster energy. WoodsSaxon single-particle wave functions were used for all the FHNC results cited.

We turn lastly to the case of the MS3 potential, and we again compare our results with others for this potential for the same two nuclei, ${ }^{12} \mathrm{C}$ and ${ }^{16} \mathrm{O}$. We note that results for these nuclei for the MS3 potential are also available within the FHNC framework using the $j j$ coupling scheme and allowing, for the first time, some state-dependence in the Jastrow correlation operator. More explicitly, the authors of Ref. [5] incorporate isospin-dependence by allowing different pairwise Jastrow functions for the protonproton, neutron-neutron and proton-neutron pairs. Otherwise the FHNC results shown in Table 5 are calculated as explained above for the B1 potential. We note again that the calculations of Ref. [5] have included the Coulomb force. The results in Table 5 have been obtained by subtracting out the cited Coulomb energy in each case. Once again, for ${ }^{12} \mathrm{C}$ our results are better than all the others. The effect of the isospin-dependence in the FHNC calculation is comparable in magnitude to the effect of the state-dependence in our calculations. However, the FHNC results [5] are less bound for ${ }^{12} \mathrm{C}$ than our TICI2 results, presumably at least in part because of their use of a spherical reference 
Table 5

A comparison of the binding energies (in $\mathrm{MeV}$ ) of ${ }^{12} \mathrm{C}$ and ${ }^{16} \mathrm{O}$ using various techniques with the MS3 potential. For a description of methods see the caption of Tables 3 and 4 . BHF is the Brueckner-Hartree-Fock method

\begin{tabular}{lll}
\hline & ${ }^{12} \mathrm{C}$ & ${ }^{16} \mathrm{O}$ \\
\hline TICI2(SI) & 46.22 & 85.56 \\
TICI2(SD) & 62.99 & 105.64 \\
FHNC/0 & & $113.5^{\mathrm{a}}[18]$ \\
& $36.5^{\mathrm{b}} / 54.2^{\mathrm{c}}[5]$ & $108.1^{\mathrm{b}} / 145.5^{\mathrm{c}} \quad[5]$ \\
FHNC-1 & & $105.3^{\mathrm{a}}[18]$ \\
& $34.7^{\mathrm{b}}[5]$ & $103.0^{\mathrm{b}}[5]$ \\
FAHT-3 (deformed) [12] & 51.4 & 107.7 \\
BHF [12] & & 118.6 \\
IDEA [20] & $44.2-44.4$ & $102.9-103.2$ \\
\hline
\end{tabular}

a State-independent 2-body Euler-Lagrange, $L S$ coupling.

b State-independent 2-body Euler-Lagrange, $j j$ coupling.

${ }^{c}$ Isospin-dependent 2-body Euler-Lagrange, $j j$ coupling.

state.

For the case of ${ }^{16} \mathrm{O}$ nucleus with the MS3 potential, the effect of the incorporation of the state-dependent correlations in our TICI2 calculations is again to increase the binding energy by about $20 \mathrm{MeV}$. Naively, one might have expected a somewhat larger increase than for the B1 potential, since the MS3 potential exploits the full V4 state dependence. Our own TICI2(SD) results are in this case close to most of the other results cited, with the exception of the isospin-dependent FHNC/0 result, where the gain in binding over the corresponding state-independent case is nearly $40 \mathrm{MeV}$. In view of the nonvariational nature of both the FHNC and Brueckner-Hartree-Fock (BHF) results, it is difficult to draw more detailed conclusions.

\section{Discussion and outlook}

We have demonstrated that the TICI2 methodology provides a very reasonable starting point for the calculation of the binding energies of light-to-medium mass nuclei. The important state-dependent pairwise correlation effects can be efficiently incorporated without destroying the variational bound. By contrast, most competing many-body methods suffer from various uncontrolled approximations, which often include the loss of a strict upper bound on the ground-state energy, even when the calculation starts from a variational framework. Such is often the case for FHNC and other more ambitious calculations performed within the correlated basis function (CBF) approach. Since such techniques provide perhaps the main competitor to our own approach outlined here, it is worth describing in some detail the source of the uncertainties that can typically arise in FHNC-type calculations.

In a nutshell, the standard FHNC formulation of Fantoni and Rosati sums all of the Jastrow cluster-expansion terms that can readily be summed by one-dimensional 
integral equations (see Ref. [5] and references cited therein for details). One drawback of this procedure is that certain cancellations between elementary exchange diagrams are thereby ignored. The resulting $\mathrm{FHNC} / n$ scheme then classifies the only omitted diagrams, the so-called elementary diagrams, according to their number of vertices or points. Thus, the FHNC/ $n$ approximation includes elementary diagrams with up to $n$ vertices. The resulting prescription is both clear-cut and leads to relatively simple integral equations which are valid for small distances. Nevertheless, the long-range parts are more problematic. In particular, for infinite homogeneous systems one neither expects to get nor obtains the correct behaviour of the liquid structure function $S(k)$ as $k \rightarrow 0$. Moreover this prescription does not allow, in principle, for correct optimisation.

In contrast to the Fantoni-Rosati FHNC/ $n$ scheme, Krotscheck has proposed a different classification which maintains the Pauli principle at each step. One possibility among several to accomplish this is to classify the exchange diagrams with respect to the number of internal lines rather than vertices. The resulting lowest-order approximant in this scheme is termed FHNC//0. In some well-defined sense it sums the union of the equivalents of the ring diagrams with a bare particle propagator and bosonic ladder terms. Effectively, for the case of infinite homogeneous systems, it sums correctly and self-consistently the most important diagrams in coordinate space when short-range correlations are important, and in momentum space when long-range correlations are important. In turn this leads to an inconsistency between the pair distribution function, $g(r)$, and the liquid structure function, $S(k)$.

From the above brief discussion it should be clear that the main difficulty and uncertainty in FHNC techniques is in the treatment of elementary exchange diagrams. We have already seen in Section 3 the differences between the FHNC/0 and FHNC-1 results, where the latter incorporates only the first-order elementary exchange diagram from the FHNC/4 set. We also note in this context that the FHNC/C scheme of Krotscheck provides another means of incorporating the omitted diagrams. Basically this scheme exploits the fact that one knows the values of these diagrams in the case of an infinite homogeneous system at momentum transfers $k=0$ and $2 k_{\mathrm{F}}$, where $k_{\mathrm{F}}$ is the Fermi momentum. The FHNC/C scheme uses these exact properties to estimate the value of the omitted terms by a simple polynomial interpolation between these limits. Thus FHNC/C is a superset of FHNC/0, but with an approximate treatment of what the latter omits. Such a correction of the FHNC/O equations is necessary for the optimisation. Unfortunately, the only place where we can numerically compare the $\mathrm{FHNC} / 0$ and the FHNC// 0 calculational schemes is for the highly unrealistic case of ${ }^{16} \mathrm{O}$ with the MT V potential, which we have already commented upon in Section 3. It has been shown [19] that inclusion of the $C$ class of diagrams has only a marginal influence.

Apart from the above difficulties and uncertainties over the treatment of elementary exchange diagrams, the incorporation of state-dependent correlations into FHNC treatments is also very fraught with difficulty. This arises essentially due to the noncommutativity of the correlation operators in a Jastrow-type product wave function when the scalar Jastrow function is replaced by an operatorial sum. The isospin-dependent correlations introduced in Ref. [5] very recently, and reported in Section 3, have led to 
workable FHNC calculations only because in this case the corresponding operators commute among themselves. The introduction of more complicated operatorial forms (e.g., of the sort used by us) will not provide such a straightforward extension. Our own TICI2 method does not suffer from these drawbacks.

We now turn briefly to the alternative IDEA approach, which is formulated purely in terms of the hyper-radius and the relative coordinate of the two particles needed to describe the pair correlations incorporated in the method. In this method, as currently practised, several uncontrolled and often unjustified approximations are made. As a consequence the results are non-variational, and usually one has a range of values depending on which of many approximations has been made, none of which is fundamentally preferred to another. A recent investigation [23] of a simple model suggests rather forcefully that even if no approximations are made, the inclusion of the hyperradial excitations in a many-body wave function that lies at the heart of the IDEA method does not provide a very cost-effective improvement. This study suggest that it would be much more efficient to include pair (and triplet, etc.) correlations to the best of one's ability, as done in the present work. From this viewpoint our own method of incorporating pair correlations is far more systematic than in the IDEA methodology.

In conclusion, our method works well for interactions and correlations of V4 form. Of course one needs a much more complicated interaction to describe phase shifts well: the latest Argonne V18 interaction has 18 components. Even though it may not be immediately obvious what kind of correlations are important when one wishes to deal with such a complicated force, it is clear that the first step is to look at interactions and correlations of the V6 form, where one includes the tensor force and tensor correlations. Such an investigation is now under way, and it appears that the methodology sketched in Section 2 can be extended to deal with such a more realistic force. Actually when we have V6 type correlations, we may already pick up the major part of the spin-orbit force as well; at this level there is no easy match between the operatorial structure of the correlations and that of the potential.

In the end we shall also have to include three-body correlations. A first indication of the importance of many-particle correlations can be seen from the case of the MS3 potential. Here the TICI2 method is no longer highly superior to state-independent many-body methods that include higher order correlations. Since, even in that case, the effect of such correlations is not expected to be very large, one might hope that inclusion of state-independent three-particle correlations is enough. This would still lead to very complicated calculations, but we believe that we can certainly deal with such a scheme. Indeed, we have already reported such results for ${ }^{4} \mathrm{He}$ using purely central (scalar) potentials [4]. Results for heavier nuclei with more realistic potentials will be reported in the future. 


\section{Acknowledgements}

This work was supported by DGICyT (Spain) grant PB92-0820, and by a research grant from the Engineering and Physical Sciences Research Council (EPSRC) of Great Britain. One of us (P.I.M.) acknowledges DGICyT (Spain) for a fellowship.

\section{References}

[1] N.S. Pudliner, V.R. Pandharipande, J. Carlson and R.B. Wiringa, Phys. Rev. Lett. 74 (1995) 4396.

[2] W. Glöckle and H. Kamada, Phys. Rev. Lett. 71 (1993) 971.

[3] A. Kievsky, M. Viviani and S. Rosati, Nucl. Phys. A 551 (1993) 241, A 577 (1994) 511.

[4] R.F. Bishop, M.F. Flynn, M.C. Boscá, E. Buendía and R. Guardiola, Phys. Rev. C 42 (1990) 1341;

Condensed Matter Theories, vol. 5, ed. V.C. Aguilera-Navarro (Plenum Press, New York, 1990) p. 253;

J. Phys. G 16 (1990) L61;

R.F. Bishop, E. Buendía, M.F. Flynn and R. Guardiola, Condensed Matter Theories, vol. 6, eds. S. Fantoni and S. Rosati (Plenum Press, New York, 1991) p. 405; J. Phys. G 17 (1991) 857, G 18 (1992) 1157, G 19 (1993) 1663.

[5] F. Arias de Saavedra, G. Co', A. Fabrocini and S. Fantoni, Nucl. Phys. A 605 (1996) 359.

[6] K. Varga and Y. Suzuki, Phys. Rev. C 52 (1995) 2885; Phys. Rev. A 53 (1996) 1907.

[7] J. Carlson and M.H. Kalos, Phys. Rev. C 32 (1985) 2105.

[8] S.C. Pieper, R.B. Wiringa and V.R. Pandharipande, Phys. Rev. C 46 (1992) 1741.

[9] I.R. Afnan and Y.C. Tang, Phys. Rev. 175 (1968) 1337.

[10] R. Guardiola, Nucl. Phys. A 28 (1979) 490.

[11] D.M. Brink and E. Boeker, Nucl. Phys. A 91 (1967) 1.

[12] R. Guardiola, A. Faessler, H. Müther and A. Polls, Nucl. Phys. A 371 (1981) 79.

[13] R.A. Malfliet and J.A. Tjon, Nucl. Phys. A 127 (1969) 161.

[14] J.G. Zabolitzky and M.H. Kalos, Nucl. Phys. A 356 (1981) 114; U. Helmbrecht and J.G. Zabolitzky, Nucl. Phys. A 442 (1985) 109; J.G. Zabolitzky, K.E. Schmidt and M.H. Kalos, Phys. Rev. C 25 (1982) 1111.

[15] E. Buendía and R. Guardiola, Condensed Matter Theories, vol. 8, eds. L. Blum and F.B. Malik (Plenum Press, New York, 1993) p. 301.

[16] S.A. Chin and E. Krotscheck, Nucl. Phys. A 560 (1993) 151.

[17] R.F. Bishop, E. Buendia, M.F. Flynn and R. Guardiola, J. Phys. G 18 (1992) L21.

[18] G. Co, A. Fabrocini, S. Fantoni and I.E. Lagaris, Nucl. Phys. A 549 (1992) 439.

[19] E. Krotscheck, Nucl. Phys. A 465 (1987) 461.

[20] R. Brizzi, M. Fabre de la Ripelle and M. Lassaut, Nucl. Phys. A 596 (1996) 199.

[21] M.C. Boscá, E. Buendía and R. Guardiola, Phys. Lett. B 198 (1987) 312; Condensed Matter Theories, vol. 3, eds. J. Arponen, R.F. Bishop and M. Manninen (Plenum Press, New York, 1987) p. 101.

[22] J.B. Aviles, Jr., Ann. Phys. (NY) 5 (1958) 251;

C.D. Hartogh and H.A. Tolhoek, Physica 24 (1958) 721, 875, 896;

J.W. Clark and P. Westhaus, J. Math. Phys. 9 (1968) 131.

[23] R. Guardiola, P.I. Moliner and J. Navarro, Phys. Lett. B 383 (1996) 243. 